\title{
Openness of Information-Communications Systems: The Rescue Tool for Preserving Information Age Heritage
}

\author{
Prof.dr.sc. Fjodor Ruzic \\ Institute for Informatics, Zagreb, Croatia
}

\author{
fruzic@open.hr
}

\begin{abstract}
Information communication systems are contextually similar to the informing science framework. In a real life environment they can be operationalized into three main categories: technology, content/information/ knowledge and user interface/ability to state information needs, to find information and to understand presented information and content. We all have information technology and digital content at our disposal yet, but what about preserving information, knowledge and overall heritage for the future generations? Today, we are confronting many problems dealing with the preservation of information age heritage in the context of the continuous technology change as well as continuous digital content explosion. The idea of openness in the world of information technology is slowly gaining acceptance throughout the information technology community. The information and digital content delivery space also needs stronger emphasis on openness in order to achieve better and wider options for preserving information age heritage. This is an urgent need because of the growing emphasis on cloud computing and networked media storages has the potential to produce mental atrophy, not only of individuals but also of entire societies. Some crucial observations as well as scope of actions and typical assumptions on this matter are also presented.
\end{abstract}

Keywords: Open system, Information-Communications systems, Heritage, Preservation

\section{Introduction}

From the first days of human history, people wanted to store information in order to establish collective memory for future generations. The history of information storage started in pre-historic time - the first stage achieved by writing or putting art on stones and walls. The process of information storage development is continuously increasing information density and the need for more and more sophisticated tools to gather and read information from various media. Hence, the electrical storage techniques of the late 19th century discarded old media formats in favor of an electrical recording. At the same time, these techniques also introduced a crucially new dimension of

Material published as part of this publication, either on-line or in print, is copyrighted by the Informing Science Institute. Permission to make digital or paper copy of part or all of these works for personal or classroom use is granted without fee provided that the copies are not made or distributed for profit or commercial advantage AND that copies 1) bear this notice in full and 2) give the full citation on the first page. It is permissible to abstract these works so long as credit is given. To copy in all other cases or to republish or to post on a server or to redistribute to lists requires specific permission and payment of a fee. Contact Publisher@,InformingScience.org to request redistribution permission. storage medium - the interface, as the way to represent and control the signal bearing data. It changed how media function. Media properties were no longer dependent solely on the data stored inside but also on the tools that provided interfaces to these data. The move to digital data and digital media software in twentieth century extended this principle further. Since almost all 
data were encoded in digital form, they could be accessed only by programmed interface. Consequently, digital media properties are now defined by the computer programs instead of being contained in the actual content.

The relations between electro-magnetic techniques developed in the second half of the nineteenth century and digital media developed 100 years later highlights some interesting points. While preceding reproduction technologies retained the original form of media, the new discarded it and an electrical signal became the base for any kind of data recording. In other words, these technologies introduced coding as a way to store and transmit media (Manovich, 2013). Further, the way we form memories in the information age is under the interaction with external information repositories, making possibilities for a new era in which we will store less information inside our brains.

Information sources interlinked through communications networks as well as human activities becoming increasingly reliant on communication have become the foundation of the information age. As Edwards, Edwards, Wahl, and Myers (2012) pointed out, we are currently in the communication age where all members of a society are connected through the Internet, not just to it. These new information technologies have decreased the need for brain power, particularly with respect to that area of the brain used to memorize stuff for a short time span. Individual memory, however, shapes the personality. Moreover, the sum of individual memories shapes the collective memory. Hence, memory becomes an important part of culture (Carr, 2011).

In the years since its start in 1992, UNESCO's Memory of the World Program has supported dozens of preservation projects intended to preserve original documents from human history. The program is designed to maintain a register of important collections from the earliest history to modern times, all of which urgently require preservation. Our entire heritage needs to be preserved in a form that will enable its consumption by future generations. And, unless an intensive international effort is made to digitize our information heritage, the knowledge we have today will fade away. Sooner or later, all old magnetic records will not be playable, even as old replay machines are disappearing. In fact, even where we have preserved collections of documents, we will not have the tools to access them. Ironically, we have found old paper to be more stable than paper from the modern times, much of which gradually loses its readability as time passes.

Solutions that involve digitization of the paper texts need to incorporate their context. This digital content is in a form such that storage of individual media or interconnected hypermedia is executed through a variety of technologies. Hence, a uniform communications system is prerequisite for fully operable open systems where Information-Communication systems provide a huge set of information that can be accessed as the information commons by anyone, anywhere, and any time. The concept of information openness is of vital interest in the information age society predicated on internationalization of the content in order to make it more accessible to more people around the world. A true Information Age society will be able to record knowledge by digital technology, and applying the open system concept, the world's knowledge becomes the legacy of our civilization. Open information technology also provides a platform for storing and distributing information. However, with the increasing digital content stored and available for sharing, the long-term preservation of the digital repositories must be assured in order to preserve our heritage for future generations.

These notions lead us to conclude that preserving the information heritage of the information age society will require a new generation of digital curators: informing professionals whose role is to preserve information in digital form for current and future use. This new class of information expert will draw insight and knowledge from the fields of information science and information technology and will help bridge the gap that has existed between the two. At the same time that information technology has given us faster, cheaper computing, improved interfaces, rapid proto- 
typing approaches have allowed the library science to develop effective systems in an efficient way.

Information science has put design and deployment of information collections at the center of its interest. In the same time, information science is currently aimed toward evaluating that content and ranking its relevance to user communities. Here we see the role played by metadata: the process of making information about information. In this process, digital assets management tools support automating metadata capture, intelligent indexing and appropriate handling of documents. With modern information technology, data capture and storing has become easier, but preservation of this has become less consistent. Without proper methods and techniques, as media degrade and interface to digital assets become obsolete, information is lost. Thus, we need an informationcommunications systems infrastructure that supports an efficient and vigorous preservation processes. Outsourcing of collective memory, through the cloud and virtual storage, could lead us to collective mental atrophy. Such scenarios lead us to more complex observations on our duty to preserve the legacy and heritage of our information age civilization.

\section{Information Age Heritage}

What marks the information age is the way that our knowledge has changed and grown - and continues to grow-with the application of new information technology. So, while we may damage the past by observing it with inappropriate information technology tools, we are still learning more and more about it. This conforms to Stille's (2002) observation that our electronic world of television and the Internet is destroying our experience from the past, and reducing our cognition to a timeless present.

\section{Nature of Heritage}

A classical interpretation of "heritage" includes all data stored in public and private repositories as well as values and knowledge that a society experienced in the past in order to preserve and transmit it to future generations. It serves to instill a common set of values and knowledge shared by individuals. It represents the social knowledge corpus in every society. How and which of these assets would be selected for saving depends upon their lasting value and significance for the entire society and its members. In the age of digital civilization, most digital assets are digital copies of pre-existing works. These digital copies are not identical to the source, but only a representation of it in digital form. The crucial part of digital heritage comes from assets that exist only in digital form, such as electronic publications, multimedia productions, cultural and scientific databases, and on-line repositories (including Web sites pages).

Further, our concept of digital heritage could be extended by considering the standard definition of heritage, which refers to values, goods and thoughts from the past, and it is represented also by cultural heritage, the legacy of tangible assets (artifacts) and intangible values of society, where such cultural heritage improves the collective memory (Craig, Graham, Kagan, Ozment, \& Turner, 2011).

There is also interesting assumption that "the dawning of the information age has created greater societal demands for information from information-gathering institutions. The information age has inundated society with vast quantities of information from innumerable sources, and technically very advanced in its transmission and storage" (McKellar, 1993, p. 349). Thus, society starts demanding more knowledge to function properly.

Information age legacy can be accessed and shared through digital objects where these new digital entities remove physical barriers to public access and enrich the knowledge of the society. The damage and deprivation of physical heritage stores in the past has meant their contents are forever lost to humanity; digital heritage has also such risk. Digital heritage can be stored in more places 
and in more forms, however, ensuring its survival in some form. This multiplicity of location and form could guarantee existence of digital heritage for future.

Existence of various digital media forms and types, as well as distributed repositories and cloud data storage with networked information, presents a challenge for digital heritage conservation. Since it is not possible to save all of the digital objects produced by an information age society, there needs to be a strong emphasis on digital heritage conservation systems that are manageable by cultural heritage professionals and information technology specialists through the open systems conceptualization. Their top priority must be to ensure proper preservation regardless of what technology is in use.

\section{Memory of the World Program}

Most of these issues related to digital heritage preservation are in some way visible through the Memory of the World Program for preserving and providing access to documentary heritage managed by the UNESCO (United Nations Educational, Scientific and Cultural Organization). The core idea of the program is that the all documented heritage throughout the world belongs to all. Thus, the international task is to preserve this heritage and also to make it permanently accessible to all. The mission of the Program is to ensure preservation of the world's documentary heritage and to assist universal access to documentary heritage by using information technology (UNESCO, 2012a). The Program maintains a register of valuable collections throughout the world that urgently require preservation. It is grounded in the belief that digital heritage needs to be preserved in a form that will allow future generations to experience it. And, unless there is intensive effort country by country to digitize this material, the knowledge we have today will vanish (Amelan, 2003).

Current issues in keeping our collective memory and digital information preservation were discussed at The Memory of the World in the Digital Age: Digitization and Preservation international conference held in Vancouver (Canada) in September 2012. It was held also with the intention of marking the 20th anniversary of UNESCO's Memory of the World Program. At the Conference, the Vancouver Declaration was adopted (UNESCO, 2012b). The Declaration asserts that digital technology offers unique means of knowledge creation and expression. It further states that the economic value of the digital information can promote national development, and supports Millennium goals. No matter what the source of this information, enabling the continuity of the heritage is the basis for good governance and effective national policies. This includes the recommendation for establishing an emergency digitization program aimed at preservation of digital assets and documentary collections that could be destroyed by natural disasters or wars, as well as an emergency forensic program for digital heritage that has become inaccessible. The Declaration also makes statements supplemental to principles in the Universal Declaration of Human Rights, asserting that each individual should have a right to information, and that national policies should promote the right to information, open government and open data. This notion denotes the open platform concepts so needed in the process of preserving heritage of information age civilization protecting us, in the same time, from the digital amnesia.

\section{How the Information Age Changes Us}

Rushkoff lays some of our possible actions with these issues. He states "In the emerging, highly programmed landscape ahead, you will either create the software or you will be the software. It's really that simple: Program, or be programmed. Choose the former, and you gain access to the control panel of civilization. Choose the latter, and it could be the last real choice you get to make" (Rushkoff, 2010, p. 1). As more and more aspects of our daily lives involve interactions with, and even dependence upon, digital technologies, we have to question what impact this dependence is having upon our collective knowledge and memory. Thus, Rushkoff (2010) calls for 
options in which instead of learning about our technology, we are establish an environment in which technology learns about us.

Information technology as well as information services have changed the way we use information sources and our memory, and they are changing the way in which our memory functions. There are assumptions that information technology, including Internet environment as communication media, has evolved to become part of a transactive memory by which we are capable of classifying information (Sparrow, Liu, \& Wegner, 2011). Such transactive memory exists in many forms, and this whole network of memory - where the individual does not have to remember everything in the world - becomes a virtual extension of human memory. Hence, the information age raises the question of how much information we have to memorize when everything we need to discover or access is in information appliances interconnected through networked information repositories. In theory, the process of relying on others to store digital information for us creates transactive collective memory that decreases individual's need, and possibly capability, for memorizing and processing information. Thus, the society could become more symbiotic with digital objects, losing the capability for knowing and understanding information in favor of knowing where the information could be found.

Thus, in the digital era information preservation awareness has become more important than ever before (Cloonan, 2012). Preservation as a managed process applies to all heritages, and in the information age (where a huge amount of digital assets are interconnected and stored in repositories) there is a need for deep understanding of what to keep for the future. These issues call for more efforts in the field of contextuality and connectivity of information repositories that maintain the heritage of our civilization.

\section{Preserving Information Heritage}

From its earliest days, our civilization has tried to find a way to store its collective memory for future generations. The history of information storage started in pre-historic times, dating from when we began putting art on stones and walls. This process has been one of continuously increasing information density. When paper and ink were first used in the process of preserving human memory, information density increased dramatically. After paper's invention, the next big step in the evolution of information preserving started with the invention of moveable type printing technology in 1440. Paper remained the key material used to store information and make it available to our collective memory, until the invention of magnetic tape in 1928 and its 1951 application to storing data.

\section{Evolution of Digital Storage}

Electro-magnetic recording and reproduction technologies represented a major departure from paper-based media. While earlier preservation technologies retained the original form of media, the new media technologies lost this connection broken by electrical signal implementation. In other words, they introduced coding as a way to store and transmit media (Manovich, 2013).

In the period from 1980 to 2000 there were three fundamental changes in techniques of digital data storing. First, the magnetic phase was followed by optical memory technologies bringing more information density and tools for storing data in various formats on compact disc (CD and CD-ROM). Second, there was introduction of electronic memory devices such as compact flash - this evolutionary step involved non-volatile and solid state memory devices that were more robust than disk drives and compact discs (which had depended upon mechanical components). It also involved the introduction of standard memory devices, such as the USB Flash Drive, that were small and removable. Third, laser technology advances produced devices that were capable of storing a huge amount of data on a single, small and versatile surface. 
At the present time, holographic memory techniques are being employed to provide information storage at high density. Holographic memory uses a light beam, instead of an electron beam, to write and read data inside crystals on ultra-thin silver plates. Its unique facility is in using the volume (space) of storage media as opposed to being limited to the media's surface. Thus, holographic memory storage is made by constructing three dimensional holograms that share the same storage medium volume. The historical evolution of storage is illustrated in Figure 1.

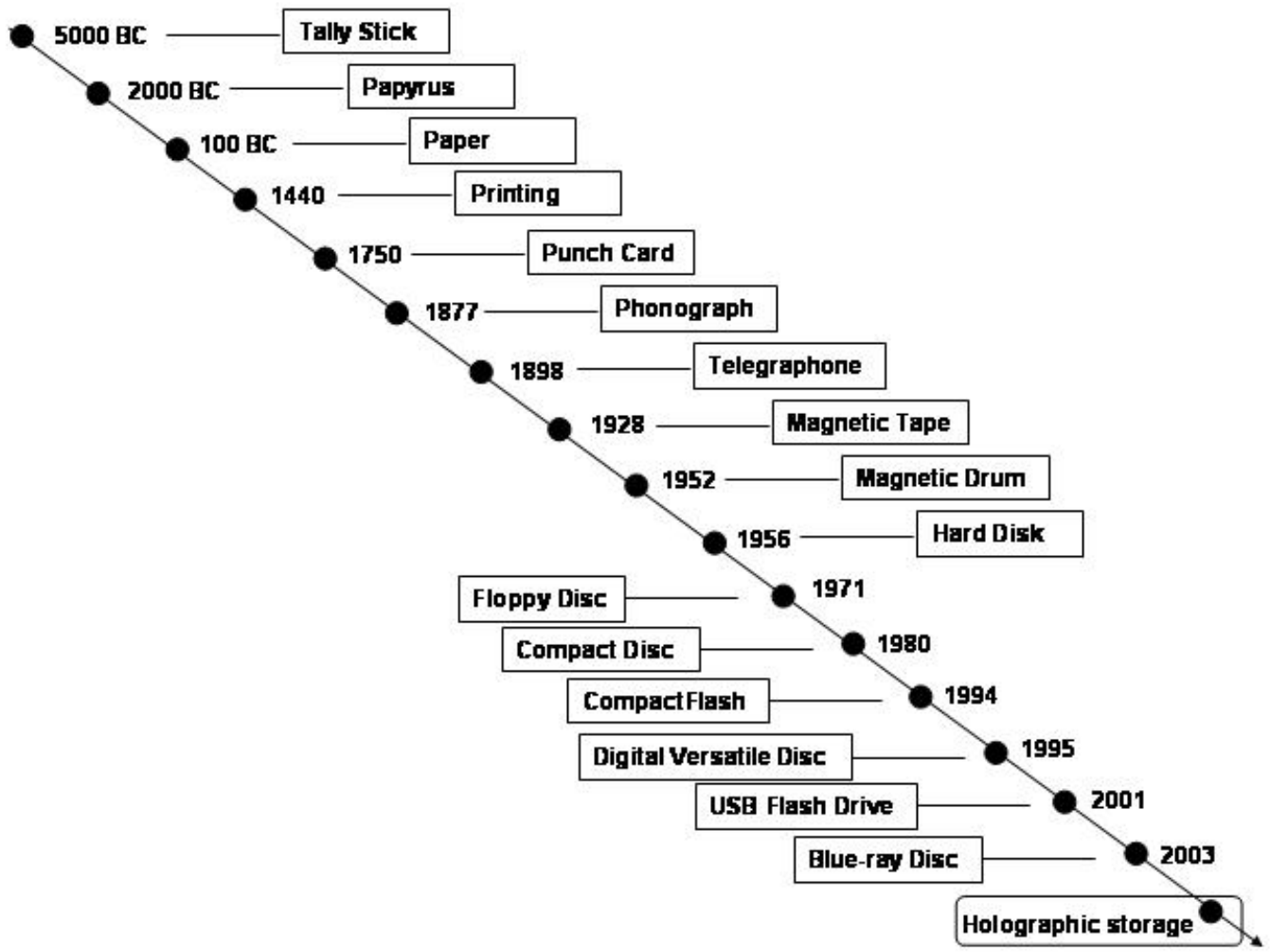

Figure 1. The timeline of information media development through the history

\section{Challenges of Digital Storage}

Among the fundamental functions of informing professionals, the preservation of information plays a crucial role. This process of information preservation depends on the nature of the materials on which much of the legacy has been stored. Information stored on durable materials can endure for a long time. Information stored on the paper can also be preserved for a long period if it was stored properly. The entire information base of human legacy from the past is in analog form, meaning that there is no need for external and additional tools to gather and read this information. Further, to preserve analog information it is quite enough to keep the media in an appropriate place under suitable conditions.

In contrast, digital preservation requires many more tasks in order to extend information storage life as well as to protect digital storage from media failure, physical loss, and obsolescence. Today, much of the information being produced is in formats that are fragile. The media on which information is stored, as well as tools needed to read the information, are under the constant threats from media corruption, hardware and software obsolescence, and loss of information context. 
Informing professionals that deal with preservation need techniques for solving the problems associated with preserving the digital heritage of our civilization. Digital preservation initially involved using digital technologies to reformat analog media to re-born digital information, and it was not concerned with the preservation of information of digital origin-information that was born digital. In the past decade, the digital preservation has broadened its objectives. The process of ensuring long-term preservation of digital objects involves questions such as which technical approach would be most appropriate for particular type of digital object. Digital preservation is more than just a technological challenge, however. It also involves organizational and social issues. It requires coping with the organization of the digital storage banks and with the procedures for maintaining the integrity, authenticity, and reliability of the digital objects. Digital objects typically involve unique hardware, operating systems and applications. What must also be captured is the ability to read interconnected stored digital objects. When these tools are missing, there is a great risk of the loss of a digital object's readability and functionality. The problems of coping with the long-term preservation of digital objects also include the ongoing need to refresh storage content in order to ensure readability in the distant future. In fact, digital objects depend upon stronger integrity than non-digital (analog) data and information. Hence, Hedstrom stated, "The challenges of maintaining digital archives over long periods of time are as much social and institutional as technological. Even the most ideal technological solutions will require management and support from institutions that in time go through changes in direction, purpose, management, and funding" (Hedstrom, 2003, p. IX).

Techniques dealing with digital preservation of digital objects from their production date to their long-term future relate to the processes of migration and emulation. Migration refers to moving digital objects from one format to another in order to maintain readability over time. Emulation is process of keeping original digital object formats and develops whatever hardware and software emulation on current hardware is necessary in order to recreate a digital environment compatible with the original object.

When standard techniques and procedures are not appropriate for digital objects preservation, digital archaeology may be necessary to acquire information from digital objects that are not accessible through currently available tools and devices. Although our civilization has its roots in the past these roots are no longer visible without archeology. The same will be true for many digital objects whose origins have been lost.

\section{Managing Digital Assets}

The information age society shows slow but steady progress towards recognizing the importance of preserving our heritage and protecting our information and knowledge repositories. However, given today's rapid technology development, society as a whole needs to become more aware of the importance of maintaining the heritage produced by the information society. Digital heritage is not just the transformation of traditional heritage into digital form. It now involves the digital media production of society and includes all forms of digital communication and digitized information/content. These new forms of artifact that carry with them both visible and invisible information relating to culture, social knowledge, and common sense. Much of modern culture is defined through digital information objects. At the same time, these objects are embedded in a networked environment so they cannot be separated from other objects or interpreted without appropriate digital context.

\section{Open Archival Information System}

It is incumbent upon every nation or group to define what artifacts to be preserved and selected from global resources available to them. This leads to the question of which digital materials should be kept for future generations. The need for this question arises from the cost associated 
with accurately selecting and preserving these objects. These costs arise because traditional preservation methods and polices are no longer usable for preservation of digital objects. Objects that are today contained in a huge number of repositories urgently need some sort of unified specification for storage, access, and manipulation. One solution involves the Open Archival Information System. This framework describes the archiving, preservation, and management of digital objects, as well as the access granted to users.

Preserving process usually consist of standardized workflows place digital materials into storage systems for subsequent management and preservation. In this process fixity of digital objects is a method for digital preservation. Digital objects have special characteristics such that after encoding additional actions are needed to verify that the encoded object is actually the same as it was prior to the encoding process, as well as throughout the preservation period. Existing methods related to compute checksums or cryptographic hashes are useful for auditing digital content in that way. To ensure the authenticity of digital copies an analog fixity check is used: a technique to check if the source content and the content of its copy are identical.

These techniques for checking that the identity of source and encoded objects relate to how Kirschenbaum (2002) defines that digital objects are the same. In his words there is a notion that one object has the same bits as another associated with the information on how those bits are physically encoded and recorded on a digital media In this process all the bits are recorded on storage media and resulted in digital objects that could also be treated as analog objects. Hence, each bit of an entire digital object could be investigated to find the accuracy of written content stored on the digital (encoding) medium.

While preservation of cultural values are core values retained in information technology retooling (Drucker, 2005), the revolution in digital media is not irrelevant in shaping the new practice. Drucker also states, "The tools of digital media are not in themselves the core, but rather what one learns from the engagement or more specifically the practice of these tools. Thus, a diverse and flexible set of skills across a range of informational, expressive, reflective and critical tasks" (Drucker, 2005, p. 246).

\section{Digital Assets Management}

Digital objects are presented by their inner structure of encoded things, and they are also allographic in contrast to analog objects. It is due to the fact that the digital objects are much richer with the details without less interesting information that are extracted from the artifact itself. The crucial task is to evaluate digital object by its long-term value in order to develop an infrastructure by which it could be preserved for a long period of time. These issues are related to Digital Assets Management.

Digital Asset Management as a content-centric system is useful as a tool for complex digital content storing, access, and reusing from various sources and technology platforms. Complex content management tools involve the integration of authoring tools for creating new digital objects with the content storage infrastructure for general and widespread use of digital assets. They are particularly relevant to the complex content challenges presented by multimedia and have been motivated by the demand for solutions to address longer-term preservation. One key initiative in the Digital Asset Management systems deployment was the Publishing Requirements for Industry Standard Metadata, a valuable specification defining a set of XML metadata for distributing, collecting and publishing content through the various system platforms. It was initiated in 1999 as a collection of computer language standards and recommendations for cataloging digital information and content transmission. It also standardizes building-block Web page language and provides an infrastructure for preserving digital content and metadata. 


\section{Challenge of Context}

There are common preservation challenges related to dealing with the context of the digital assets. Contextualization of content in collections is an important aspect of the past and current work of informing professionals. Besides informing science professionals, however, there are many groups and communities of users that have documented their history and life experience both digitally and though other artifacts. Thus, preserving context is a great challenge to any preservation activities involving digital assets. This notion could be found in the words of Sterling (2009) stating that such a task is aimed at protecting our legacy while ensuring its importance to the present and future.

Cook (1993) has advocated the emergence of a new archival paradigm called the post-custodial age in which he proposes that informing professionals should move from a content-based history to a context-based future. Changing from passive custodians to active document analysts, informing professionals have to evaluate digital objects in terms of context, function, interrelationships, and creation. Cook's concept of a postcustodial age emphasizes the skills of the informing professionals and it is timely in its relevance to the new challenges of information age society. These emerging professionals have to emphasize their expertise in contextualizing digital assets available through globally interconnected information sources and repositories.

The newly created digital memory of our civilization involves the development and proposal, through a digital reconstitution of physical channels and media, of a new social picture of the past in which previously unknown visions of contemporary culture are concentrated and expressed. A historic perspective is essential in this process. "Its foundations were laid with the rise of an archival vision of the world from which documentation, conservation and museology have emerged as fully fledged disciplines" (Renaud, 2002, p. 11).

Many problems dealing with how information technology is used in information repositories currently exist. These problems center around how to secure digital objects through the time in which they are accessible in its original form. Information technology is constantly changing and quickly develops incompatibilities with its predecessors. Such a scenario creates the possibility of digital objects inaccessibility within just a near future. There is also the matter of Internet as a whole - the widespread publishing medium of the information society - and some argue that all Internet content should be saved since the Web pages, forums, blogs etc. present (in some way) the picture of the information age society and that such content creates new form of human heritage.

When individual and social knowledge are digitized into some interoperable defined structural forms, there remains the possibility that a portion of the overall heritage will be still unknown. Liu (2004) concludes that the core problem is the ethos of the unknown, the place of the unencoded, unstructured, and unmanaged. In our current age of knowledge work and total information, there is a question about how to convey our incomprehensible experience in structured media of knowledge. For him it could be the arts. Hence, we find the interoperability as another key category in preserving processes. Interoperability as a term evolved over the past years from device interoperability, inter-service interoperability, to system interoperability that includes unrestricted sharing of resources between different systems. This can refer to the ability to share data between different components or it can be defined as the exchange of information and resources between different computers through communication networks.

To some extent, there are forms of emotional embodiment of technology in current humancomputer interfaces (Hansen, 2004) conceptualized through open-ended recursivity of technology and human interaction (Hansen, 2006). Hansen (2006) also argues for coevolution of man and machine, making electronic literature and digital art new kinds of intermediations. These issues introduce new waves of making cultural heritage - waves that are extremely dependent on tech- 
nology that evolves very quickly and are beyond corresponding preservation capabilities as they currently exist.

Current communication tools offer the possibility of capturing the richness of our cultural diversity - but the way we manage and disseminate digital information will make the difference. Abid \& Radoykov (2002) advanced the assumption that for cultural institutions traditionally assigned with the collection and preservation of cultural heritage, these new trends open to them new questions relating to what digital artifacts should be kept for the future and how they should be preserved. Under this assumption, future generations of online digital collections should work with emerging groups of users in the development of concepts and content in order to be able to create intelligent user interfaces and to give meaningful information (Cameron, 2001).

\section{Open Systems Paradigm}

The idea of openness in information-communications systems provides new opportunities and challenges in the field of digital assets preservation. Hence, informing professionals should develop system for openly accessible digital content that will foster the exchange of ideas and knowledge of the entire memory of a civilization.

Openness as a concept crucially influences preservation of information age heritage. It is related mostly to infrastructure, openness of information systems, and knowledge. Thus, it provides informing professionals with a huge number of possibilities. In such a way, Interoperable Open Architecture was developed in order to accomplish performance, scalability, and reliability synergy. It is based upon open standards that deliver interoperability among systems and applications built at different platforms. These open standards allow interoperability using common System Data Dictionary as a meta-data collection describing the semantic meaning of information within intersystem data exchange. This semantic content gives the context by which applications could access relevant data in information processing. Naturally, the context is needed to understand the content and to confirm meaning of the accessed data. The goal is to make systems working upon common semantic models fully interoperable.

\section{Definitions of Open Systems}

In the field of open systems two approaches and definitions of open systems coexist.

Computing-based open system refers to a set of open programming code, interfaces, and standards allowing any user of one system to operate and communicate with any other system. In the past, the term open source was applied only to the source programming code. In recent times, it is often applied to other forms of deployment such as open system ecology, and open technology including open hardware, open software, open content, and open access. Open-technology combines hardware, software, and content in a way that breaks barriers between technology and information produced and accessed by the same technology. This open system based culture facilitates multiple forms of different platforms, approaches and goals of system development and deployment.

System-based open system refers to flexible systems that can adapt and change by interacting with their external environment. It is in fact close to the thermodynamic system that exchanges its characteristics with the environment. The second law of thermodynamics tells that a system has entropy. In process of open system development the task is to remove the entropy from the system. Hence, we find the roots of the theory of open systems. The open systems theory is crucial in understanding a living organism and systems, and it states that living systems are open systems due to their characteristics of interaction with the environments. The theory of open systems belongs to the upper level of the general system theory that describes the general principles of any system. 


\section{Interactions with Open Systems}

Besides these two core description of open systems, there is an issue related to the interaction of a system and society in a form of an open system as a process in which man and technology exchange information with their environment. In the context of open systems, an interesting perspective involves characterizing organizations as a system according to several perspectives. Thus, Scott (Scott, \& Davis, 2007) stressed three perspectives on organizations: Organizations as Rational Systems, Organizations as Natural Systems, and Organizations as Open Systems. Open systems perspective is crucial in transforming our view on digital heritage that would be preserved for a long time. Bertalanffy (1956) stated that open system theory was initially developed by defining the concept of a system in which existed structure provides interdependence of individual parts. Bertalanffy also argued that the open system is strongly connected with its environment. Thus, the changes in the environment could start a process of changing in the system. In the same time, a change in the system could produce the environment change. This notion of the open system has also influenced the study of living systems, including groups and large social systems.

Bertalanffy (1950) found that living (organic) systems are open systems since they exchange materials and other components with environment, with constantly changing states of their components. Hence, Bellinger (2004) also stated an organization as open system characterized by interrelationships among the parts of the system and parts from external environment. The system approach has therefore been applied to the study of organizations, assuming that the environment surrounding the organization exerted important effects on its structure, and the organization similarly affected its environment. The organizational behavior and its complexity are covered by academic research contending that the open organization reacts quickly to current change, and exploits resources more successfully than closed organizations (Ahmad, Veerapandian, \& Wee, 2011).

\section{Opening up Information-Communications Systems towards Preserving Digital Heritage}

New media tends to be strongly emphasized in Information-Communications systems deployment and almost always correlates to digital media. In fact, some theorists explicitly define "new media" in terms of computer and communications use. For example, Manovich (2001) defined new media by modern media and computers convergence based on five principles: numerical representation, modularity, automation, variability, and cultural transcodification. Manovich's fifth principle describes the social effects of computer whereby software mediation creates a new digital culture.

\section{Informing Systems and Information-Communications Systems}

Very similar to the concept of Information-Communications systems slightly developed from the beginning of the 1980s, informing systems comprise three vital constitutional components. Cohen (1999) stated that informing systems consist of three components:

- Informing environment - the system components of the system on the informer's and sender's side.

- Delivery system - the combination of technological and non-technological elements including the communications channel

- Task completion system - the components of the system related to the user and receiver of the information. 
Thus, information-communications systems also have three core categories: the content (digital objects), communications (channels and media), and interfaces (information appliances). Information-Communications systems also enable interactive extended communication-a form of a discourse network. Liu (2004) describes a discourse network as the network of technologies and institutions serving the entire society in the selection, storing, and processing relevant data. In such way, the society creates new media based information age heritage.

\section{The Impact of New Media}

From the viewpoint of information technology, the current information-communications systems converge with media - this convergence is about communications - services/interfaces - content triangle. Thus, we are confronted with new form of media, referred to as digital, which is reshaping not only media industry but also the cultural milieu of regions, nations and the globe. The new smart devices, linked through communications systems, are creating new forms of information-communications systems. The new form of information appliances and ubiquitous information technology creates the basis for the concept of an information-processing utility. Based on interactive and ubiquitous carriers of information, the first generation of new informationcommunications systems evolved to provide easy communication over time and space barriers. These new information-communications systems are media. They are virtual communication spaces for groups and societies interested in the exchange of information and knowledge in a global environment. Further promising technologies are pervasive computing and augmented reality. The notion of pervasive computing is a convergence of the Internet and mobile technologies. The emergence of communications networks in which smart devices communicate with each other and with the rest of the system opens the new forms of information processing performed by smart devices. Hence, we are entering the era of new media that are in fact, tools that transform our perception of the world and in turn render it invisible or visible just as culture does.

In this process of entering information-communications systems and new media into our society, culture and collective memory, there is also a notion that it is driven by technology and culture convergence. When society adopts the concept of openness, the technology used within open society should be also based on the openness. Hence, information-communications systems comprising new digital media making new form of heritage is under the process of developing openness. This process is needed for all basic components making information-communications systems functional and usable by all members of entire information society. The following text describes and presents how the concept of openness should be implemented in basic components of information-communications systems.

\section{Open network}

The Web as a platform promotes the digital revolution and democracy (in some ways). Like democracy itself, it can afford certain kinds of protections. In the context of overall open systems democracy, the Internet neutrality is one of the core issues - one that needs to be preserved in order to allow the unrestricted development of the Web, based on its principles of universality and de-centralization (Berners-Lee, 2010). In this process, open standards are the key to promoting innovation and creativity of Internet use and in creating the diverse assets of Web sites. The open principle within network ecosystem design thus includes the principle of universality by which every part of the network, information sources and the content has universal identification and the information flow is not dependent upon specific technology.

Does information age formalization leads to an Internet civilization? Raising this question offers insight into motive for retaining an eternity of information sources and their digital assets. However, there is no yet adequate storage method for digital assets that can keep recorded content for a long time. In the information age society digital assets are stored through interconnected infor- 
mation repositories, and with the vulnerability of information technology we may lose a huge set of information making our knowledge partial. In the near past, information professionals predicted the inter-connected information environment well in advance of actual Web use. These activities were done through mapping and indexing associations between ideas, facts, and documents very similar to the way in which information is indexed by the human brain. Putting them in the context of the Web environment we may find new solutions in the concept of a Semantic Web.

The Semantic Web includes a concept known as a Web of Linked Data, enabling the automated or semi-automated querying, sharing, and interpretation of data from distributed sources in heterogeneous formats (Shadbolt, Hall, \& Berners-Lee, 2006). The basic building blocks are the Universal Resource Identifiers (URIs), which denote pieces of information and context. In such way, information can be connected with new contexts, and differently reused. Thus, the Web becomes the ubiquitous hypermedia system.

\section{Open interface/software and presentations}

Computers, the Internet and information appliances have changed the way we live. Nevertheless, most of the technology is constantly changing, producing diverse platforms and solutions. It is hard to handle them properly and beckons for alternative methods of technology development. As a solution we could apply the open source concept, which is not a technology, but rather ecology defined through a collaboration that has evolved along with the Internet. Open source software as a system component is related to source code openness attributes, including free modifications, development and additional design. The production of software by open source communities is the core of the same relationship that exists between solutions and open source ecosystems, respectively. The open structure can be viewed as a platform for ecosystems, built around open standards, and transparency. Understanding the dynamics of an open source ecosystem is actually complex task but it is obvious that open-structure represents the heart of an open source ecosystem's infrastructure. The crucial element here is transparency. Transparency is the core category of any successful open source project providing us visibility into the code and the open source community.

Just like the open-source software initiative and concept that is widely used today, there is also open-source hardware concept - the design as well as the code that runs on the designed product is open, allowing others to use source design. It strongly influences media devices and the user interface ecosystem. The goal is to deploy complete and universal user platforms based on openness of technology in order to provide user with helpful tools for acquiring knowledge, media consumption and learning for the future.

\section{Open content and access}

The open system concept also includes an open access category. The open access category denotes the manner of access to the information technology and to the digital content residing in networked information depositories. In this context, digital content describes any individual media or interconnected hypermedia where the interconnection is executed through a variety of technologies. Hence, the uniform communications system is prerequisite for fully operable open access scenario. Recently it has been provided by the Internet, allowing users to communicate and to gather information. Such open access systems provide a huge set of information commons available to anyone, anywhere, and any time. It is close to the univerzalization of access, which is needed to avoid multidimensional gap separating those who have and those who do not have universally accessible technology.

Open content enables new forms of creating and accessing information and knowledge. Information age society relies heavily on international interactions. Hence, information and knowledge content openness empower internationalization of the content making it more accessible to more 
people around the world. With open content more people are able to access more information repositories, improving collective knowledge and memory. This open content is connected with the new open ecosystems through the information sharing tools as a means by which new collective knowledge is acquired.

The open access model is, to some extent, accepted as a publication process but is mainly oriented towards the preservation of institutional digital materials. It includes open informing activities dealing with the freedom to access, study, and understand information in order to develop knowledge. Such freedoms depend upon further efforts directed towards finding ways to foster open content publishing and protection of digital resources. The potential of open access and its opportunities will depend upon developing new practices in information storing and distribution that are not as restrictive as those encountered in the era of print media.

The information age society is able to record knowledge through digital technology, and with the open access concept the knowledge becomes open and free. However, with the increasing amount of digital content stored and available for sharing, the long-term preservation of the digital repositories must be assured in order to preserve our heritage for future generations.

\section{Open Crowdsourcing}

Crowdsourcing is a process involving individuals independently providing their efforts and ideas towards accomplishing a common task. Although there are currently not many cases of applying crowdsourcing in heritage preservation activities, it is obvious that the real potential of crowdsourcing exists.

In an open platform community crowdsourcing is not a new concept. However, it is still not a well-established term and category as applied to the preservation of digital assets. Most crowdsourcing projects in heritage institutions have not involved large numbers of individuals. With the Internet and Web tools employed as a highly interactive and participatory platform there is a room for developing crowdsourcing initiatives. Heritage organizations need to play a central role in establishing initiatives that connect individual users to the broader task of preserving their digital assets.

From the perspective of functionality, there is a notion that crowdsourcing is mostly based on synergy, serving to amplify the effects of the individual parts or team members. Synergy in such way actually redirects intellectual power from objects to the relationships between team members, and to the effects produced by these relationships. Corning (1983) proposed that synergy influenced the evolution of cooperation and the evolution of complex systems. We can see these cooperative interactions everywhere around us, and synergy combines functional effects of the parts interacted in an open system.

It is a known fact that the Internet changes our living habits and that it offers opportunities for new kinds of creativity and problem-solving scenarios. Interconnected individuals and groups can create new participation forms and sharing based on facilities that information resources provide (Shirky, 2010). When the openness and universality of the Web meet increased openness and universality in the domain of information age heritage preservation, the potential of crowdsourcing will grow. The digitization of analog heritage resources means that heritage organizations will increasingly use the Web as a platform for the integration of heritage artifacts. Digitization also ensures long-term preservation of the information, especially those held on dedicated media. It creates new ways of access heritage content. When information is in a digital form and part of an open network, there are many possibilities for cultural artifacts to be shared (Oomen \& Aroyo, 2011).

The Crowdsourcing Representation Information to Support Preservation (cRISP) program initiative was established as a collaborative approach in using Web archives for collecting representa- 
tion information to ensure long term access to digital content where representation information is essential for successful rendering of digital content in the future (Pennock, Jackson, \& Wheatley, 2012). It is aimed toward integration of crowd knowledge by which online sources of Representation Information would be found, collected, and preserved.

\section{Discussion}

The value of an information entity in the information age depends on its role in social mobilization and its circulation through communications networks providing information delivery in digital form. These activities are producing new informational affairs which create the need for understanding how to install them within the information age culture. This is the task that we must do today in order to preserve our knowledge and memory for everlasting time.

Media is shaped by two core categories - time (as short as possible) and space (as long as possible). These changes have led to the information society as we know it today. But processing technology development is maturing and the information society continues to change. Communication is increasingly becoming the vehicle for our information processes, moving us towards a new form of communication society. Communication has been with us since the earliest days of our civilization and it is the tool that has driven all our evolutionary phases. The new media reshapes information processing into virtual activity — one that is based upon virtual artifacts that often disappear after an information-communication activity is finished. What will the future civilizations find about our information society if they must rely upon inaccessible digital information assets and virtual artifacts?

We need adequate techniques and tools for preserving the digital heritage of information and knowledge society. Traditional museum, libraries, and similar institutions are not well suited to coping with the digital heritage, and there is a pressing need for digital repositories of the whole social knowledge corpus. These new knowledge/memory institutions for the digital society will preserve the digital matrix of the information society alongside traditional archives, libraries, and museums. Preserving digital heritage is a joint public and private responsibility where specific and well-developed heritage institutions exist for specific types of culture, government, and scientific information. These institutions are well versed for preservation of non-digital assets. Further, the term digital is a characteristic of almost all forms of cultural expression in the information age, well beyond the boundaries of traditional forms of culture. To preserve these broader aspects of the modern digital information world, we need a new type of heritage institution and we must take actions encouraging the establishment of digital heritage repositories. To some extent, this portion of heritage preservation activity could be enhanced through crowdsourcing efforts derived from the open platforms conceptualization and open source ecosystems.

Each society has its collective knowledge that is product of technology and culture. When information technology is acting as the bearer of social knowledge, information technology professionals play an important role in the process of recognizing knowledge as asset that is the product of interconnected nodes of information, people, tools, and social norms and values. Thus, every society has to find its own culture portfolio residing in information technology use. Technology should be accepted in accordance with the entire cultural values and beliefs of society and its members. Every individual with his or her learning models and acquired knowledge brings his or her share to knowledge corpus of the whole society. All of these create new digital society heritage in which involves every digitally connected individual. With the development of sciences, technology and economy of countries, and the advancement of people's cultural level, many countries slowly but surely place more emphasis to the preservation of their cultural heritage. The whole society recognizes the importance of preserving human heritage and maintaining our intellectual base. However, with rapid technology development, we are in front of the new need to conserve our heritage within new information society. In the context of Internet and social net- 
works, every piece of information is incorporated into digital objects. Digital objects of a specific type are embedded in a networked environment and cannot be separated from other object types. Digital objects have become the new atoms, the building blocks of information society. To preserve this aspect of the modern world, we need a new type of heritage institution and new forms of activities, tools, and techniques capable of performing this task successfully. The artifacts from past civilizations were analyzed by history, archeology, sociology and policy sciences, providing us with wisdom about our past. But, can this stream exist in future after our civilization dominated by information technology no longer exists?

\section{Conclusion}

As digital information dissemination and storage has become easier, the preservation of digital objects has become less reliable due to shorter digital media life. As media degrade and interfaces to digital assets become obsolete, information is lost. We need an information-communications systems infrastructure that supports an efficient and vigorous preservation process. This digital outsourcing of collective memory, through the cloud and virtual storage, could lead us to collective mental atrophy. Such a scenario further motivates our duty to preserve the legacy and heritage of our information age civilization.

Any organization or society trying to maintain continuity through keeping its entire knowledge corpus must capture the critical knowledge of each individual and to ensure the transfer of that knowledge to successor generations. The key to knowledge continuity is preserving the relationships between knowledge elements and building a network to enable communication. Hence, each society has to find the ways to establish open networks, open content and open interactions in order to establish the base for everlasting preservation of its digital heritage, ensuring that heritage will remain for future civilizations as well.

\section{References}

Abid, A., \& Radoykov, B. (2002). Access and preservation in the information society. Museum International, 54(3), 64-72.

Ahmad, K. Z., Veerapandian, K., \& Wee, Y. G. (2011). Person-environment fit: The missing link in the organizational culture -commitment relationship. International Journal of Business \& Management, $6(11), 11-20$.

Amelan, R. (2003). Memory of the world: The key to diversity. The New Courier, 2, 28-31.

Bellinger, G. (2004). Victims of the system or systems of the victim. Retrieved from http://www.systemsthinking.org/vossov/vos.htm

Bertalanffy, L. (1950). The theory of open systems in physics and biology. Science, 111(2872), 23-29.

Bertalanffy, L. (1956). General system theory. General Systems, 1(1), 1-10.

Berners-Lee, T. (2010). Long live the Web: A call for continued open standards and neutrality. Scientific American, 303(6), 80-85.

Carr, N. (2011). The shallows: What the internet is doing to our brain. London: W. W. Norton \& Company.

Cameron, F. (2001). Wired collections - The next generation. Museum Management and Curatorship, 19(3), 309-315.

Cohen, E. B. (1999). Reconceptualizing information systems as a field of the transdiscipline informing science: From ugly duckling to swan. Journal of Computing and Information Technology, 7(3), 213-219.

Cloonan, M. V. (2012). Preserving our heritage: perspectives from antiquity to the digital age. New York, NY: Neal-Schuman Publishers. 
Cook, T. (1993). The concept of the archival fonds in the post-custodial era: Theory, problems, and solutions. Archivaria, 35, 24-37.

Corning, P. A. (1983). The synergism hypothesis: A theory of progressive evolution. New York, NY: McGraw-Hill.

Craig, A. M., Graham, W. A., Kagan, D., Ozment, S., \& Turner, F. (2011). The heritage of world civilizations: Combined volume (9th ed.). Upper Saddle River, NJ: Pearson Prentice Hall.

Drucker, J. (2005). Humanities games and the market in digital futures. Criticism, 47(2), 241-247.

Edwards, C., Edwards, A., Wahl, S. T., \& Myers, S. A. (2012). The communication age: Connecting and engaging. London: SAGE Publications.

Hansen, M. B. (2004). New philosophy for new media. Cambridge, MA: MIT Press.

Hansen, M. B. (2006). Bodies in code: Interfaces with digital media. New York, NY: Routledge.

Hedstrom, M. (2003). It's about time: Research challenges in digital archiving and long-term preservation. Final report workshop on research challenges in digital archiving and long-term preservation. Ann Arbor, MI: University of Michigan School of Information.

Kirschenbaum, M. G. (2002). Mechanisms: New media and the forensic imagination. Cambridge, MA: MIT Press.

Liu, A. (2004). Transcendental data: Toward a cultural history and aesthetics of the new encoded discourse. Critical Inquiry, 31, 49-84.

Manovich, L. (2001). The language of new media. Cambridge, MA: MIT Press.

Manovich, L. (2013). Media after software. Journal of Visual Culture, 12(1).

McKellar, S. (1993). The role of the museum archivist in the information age. Archivaria, 35, 347-352.

Oomen, J., \& Aroyo, L. (2011). Crowdsourcing in the cultural heritage domain: opportunities and challenges. In M. Foth, J. Kjeldskov, \& J. Paay (Eds.), Proceedings of the 5th International Conference on Communities and Technologies. 29 June - 2 July, Brisbane, Australia, pp. 138-149. New York, NY: ACM.

Pennock, M., Jackson, A. N., \& Wheatley, P. (2012). CRIsp: Crowdsourcing representation information to support preservation. In R. Moore, K. Ashley, \& S. Ross (Eds.), Proceedings of the 9th International Conference on Preservation of Digital Objects, iPress 2012 (pp. 17-20). Toronto: Faculty of Information.

Renaud, A. (2002). Memory and the digital world: A few philosophical pointers for new memory practices in the information era. Museum International, 54(3), 8-18.

Rushkoff, D. (2010). Program or be programmed: Ten commands for a digital age. New York, NY: OR Books.

Scott, R. W., \& Davis, G. F. (2007). Organizations and organizing: Rational, natural and open systems perspectives. Upper Saddle River, NJ: Pearson.

Shadbolt, N., Hall, W., \& Berners-Lee, T. (2006). The semantic web revisited. IEEE Intelligent Systems, 21(3), 96-101.

Shirky, C. (2010). Cognitive surplus: Creativity and generosity in a connected age. New York, NY: Penguin Press.

Sparrow, B., Liu, J., \& Wegner, D. M. (2011). Google effects on memory: Cognitive consequences of having information at our fingertips. Science, 333, 776-778.

Sterling, B. (2009). Revisions of digital culture. In S. Krier (Ed.), Me you and everyone we know is a curator. Breda: Graphic Design Museum. 
Stille, A. (2002). The future of the past: How the information age threatens to destroy our cultural heritage. London: Picador.

UNESCO (2012a). Memory of the world: The treasures that record our history from 1700 BC to the present. London: HarperCollins Publisher.

UNESCO (2012b). The memory of the world in the digital age: Digitization and preservation. UNESCO/UBC Vancouver Declaration. Paris: UNESCO Publishing.

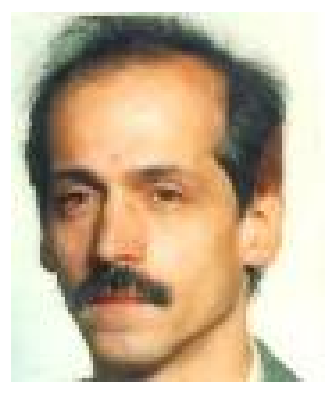

\section{Biography}

Fjodor Ruzic is doctor of information sciences at University of Zagreb where he is lecturer in new media, and interactive multimedia systems. His recent research activities cover the integration of information contents and integration impacts on development of information theory and praxis. He was working on both research and implementation sides of networked databases, educational material, and digital media as well as on information-communications systems development. He published over 130 scientific and research papers in international journals and he is author of several books on graphical user interfaces, multimedia and Internet.

Current work is also related to contextual and cognitive Web development. He promotes Open System thoughts in information technology community and he is author of national Open Source Policy. Besides these activities, he was involved in enacting Electronic Sign Act and The Law on Electronic Document as the author of their final texts. He promoted ECDL system of excellence for office workers in public administration as licensed ECDL examiner. Internationally, he participated in preparation of the Recommendation on e-democracy within Council of Europe and especially on e-governance principles. 